\title{
Identification of Bacterial and Fungal Communities in the Roots of Orchids and Surrounding Soil in Heavy Metal Contaminated Area of Mining Heaps
}

\author{
Miroslav Böhmer ${ }^{1,2, * \mathbb{D}}$, Daniel Ozdín ${ }^{3}$, Matúš Račko ${ }^{3}$, Michal Lichvár ${ }^{4}$, Jaroslav Budiš ${ }^{2,4,5}$ \\ and Tomáš Szemes $1,2,4$ \\ 1 Department of Molecular Biology, Faculty of Natural Sciences, Comenius University in Bratislava, \\ Ilkovičova 6, 84215 Bratislava, Slovakia; tomas.szemes@uniba.sk \\ 2 Comenius University Science Park, Ilkovičova 8, 84104 Bratislava, Slovakia; jaroslav.budis@gmail.com \\ 3 Department of Mineralogy and Petrology, Faculty of Natural Sciences, Comenius University in Bratislava, \\ Ilkovičova 6, 84215 Bratislava, Slovakia; daniel.ozdin@uniba.sk (D.O.); matus.racko@centrum.sk (M.R.) \\ 4 Geneton Ltd., Ilkovičova 8, 84104 Bratislava, Slovakia; michal.lichvar@geneton.sk \\ 5 Slovak Center of Scientific and Technical Information, Lamačská Cesta 8/A, 81104 Bratislava, Slovakia \\ * Correspondence: bohmer6@uniba.sk
}

Received: 12 September 2020; Accepted: 19 October 2020; Published: 21 October 2020

\begin{abstract}
Orchids represent a unique group of plants that are well adapted to extreme conditions. In our study, we aimed to determine if different soil contamination and $\mathrm{pH}$ significantly change fungal and bacterial composition. We identified bacterial and fungal communities from the roots and the surrounding soil of the family Orchidaceae growing on different mining sites in Slovakia. These communities were detected from the samples of Cephalanthera longifolia and Epipactis pontica from Fe deposit Sirk, E. atrorubens from Ni-Co deposit Dobšiná and Pb-Zn deposit Jasenie and Platanthera bifolia by $16 \mathrm{~S}$ rRNA gene and ITS next-generation sequencing method. A total of 171 species of fungi and 30 species of bacteria were detected from five samples of orchids. In summary, slight differences in $\mathrm{pH}$ of the initial soils do not significantly affect the presence of fungi and bacteria and thus the presence of the studied orchids in these localities. Similarly, the toxic elements in the studied localities, do not affect the occurrence of fungi, bacteria, and orchids. Moreover, Cortinarius saturatus, as a dominant fungus, and Candidatus Udaeobacter as a dominant bacterium were present in all soil samples and some root samples. Finally, many of these fungal and bacterial communities have the potential to be used in the bioremediation of the mining areas.
\end{abstract}

Keywords: Orchidaceae; soil; bacteria; fungi; microbiome; heavy metal; NGS

\section{Introduction}

Orchids belonging to the family Orchidaceae are well known for their rarity and presence of mycorrhizal associations [1,2]. This specialization contributes to the diversity and rarity of orchid species $[3,4]$. They grow in specific habitats such as mining dumps. Mine heaps and mine wastes created by the mining industry are one of the extreme habitats made by anthropogenic activity. Interestingly, mine heaps create an environment with specific ecological conditions for plant adaptation. They are characterized by the lack of soil as well as nutrients and moisture, and the absence of a humus layer [5]. Unique species from the family Orchidaceae, such as narrow-leaved Helleborine (Cephalanthera longifolia; Figure 1D) and Pontic Helleborine (Epipactis pontica; Figure 1E) which grows on the Fe deposit in Sirk, dark-red Helleborine (E. atrorubens; Figure 1C) from the Ni-Co deposit Dobšiná and Pb-Zn deposit Jasenie (E. atrorubens; Figure 1A) and Lesser Butterfly orchid (Platanthera bifolia; Figure 1B) from the $\mathrm{Pb}-\mathrm{Zn}$ deposit Jasenie which grows on the initial soils (rankers) and tolerates high concentration of 
heavy metals, are well adapted to extreme conditions of the environment. These organisms germinate only after colonization with a suitable mycorrhizal fungus [6].
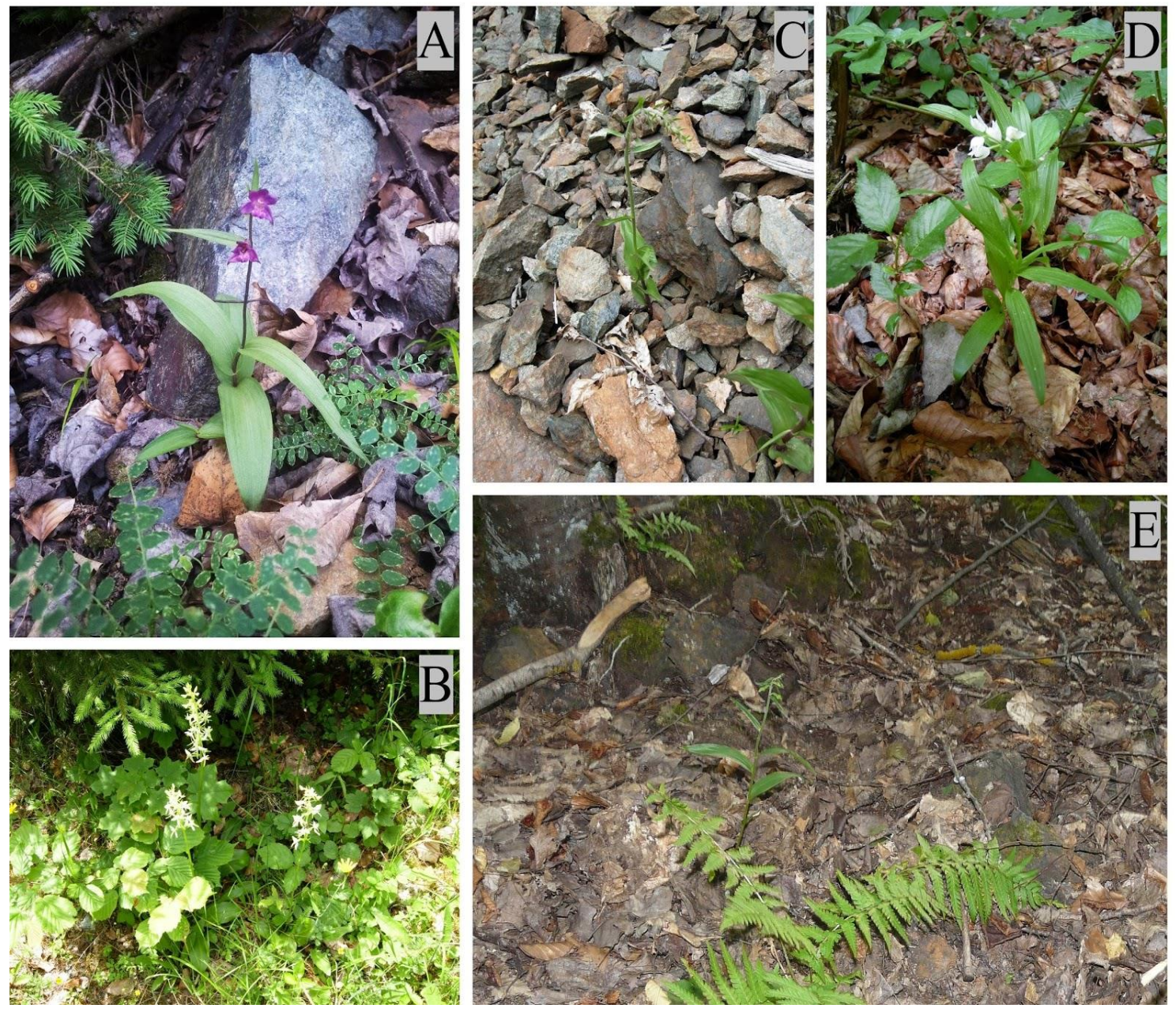

Figure 1. Unique species from the family Orchidaceae. E. atrorubens (A) and P. bifolia (B) from the $\mathrm{Pb}-\mathrm{Zn}$ deposit Jasenie, E. atrorubens (C) from the Ni-Co deposit Dobšiná, C. longifolia (D) and E. pontica (E) from Fe deposit Sirk.

The fungi provide the basic physiological function for the orchids in the uptake of needed nutrients and water, otherwise inaccessible to plants [7], facilitate the emergence and survival of vegetation under stressful conditions [8], stabilize waste material through a network of veneers and improve its structure by producing substances that bind to soil particles and lead to the formation of soil aggregates [9].

The microorganisms are known to detoxify metals by several mechanisms including ion exchange, chelation, adsorption, crystallization, valence transformation, extra- and intracellular precipitation and active absorption [10]. The sorption and accumulation of metals depend on various factors such as $\mathrm{pH}$, temperature, organic matter, ion species and the presence of other ions in solutions that may be in competition. They develop adaptation by temporarily altering their developmental patterns or modifying physiological characteristics depending on the toxicity of metals, which is affected by the concentration and form of salts in which the metal exists [11].

Bacteria play a key role in the element biotransformation, biogeochemical cycling, metal and mineral transformation, biological weathering and formation of the soil and sediment [12]. They have high importance in industrial processes, bioremediation, and heavy metal tolerance [13].

To identify fungi and bacterial communities the Illumina MiSeq platform was used. It is a scalable, ultra-high-throughput, cost-effective and a powerful tool to detect soil microbial communities at 
greater depth and with more detailed sequence information $[14,15]$. It has been successfully applied to identify microbial communities almost everywhere $[16,17]$, including heavy metal contaminated environments [18,19].

The aim of this paper is to identify the composition of bacterial and fungal communities present in the roots and the surrounding soil of the orchids growing on heavy metal contaminated areas of mining heaps. To detect these communities we used 16S rRNA gene and ITS next-generation sequencing approach. At the same time, our aim was to compare the data of fungal and bacterial communities with the $\mathrm{pH}$ value in the $\mathrm{Pb}-\mathrm{Zn}$ mining heap Jasenie-Soviansko, Ni-Co mining heap Dobšiná-Dobrá nádej and Fe mining heap Sirk-Železník. The broader goal of the study was to determine the unique microbial content of plant root collected from contaminated soil as potential biomarker applicable in further studies or monitoring efforts.

\section{Materials and Methods}

\subsection{Site Description}

Plants, soils, and geological bedrock investigated in this study originated from three abandoned mining sites in Slovakia, Central Europe ( $\mathrm{Pb}-\mathrm{Zn}$ deposit Jasenie-Soviansko, Ni-Co deposit Dobšiná-Dobrá nádej and Fe deposit Sirk-Železník).

The $\mathrm{Pb}-\mathrm{Zn}$ deposit Jasenie-Soviansko (N $\left.48^{\circ} 89^{\prime} 27^{\prime \prime}, \mathrm{E} 19^{\circ} 44^{\prime} 15^{\prime \prime}\right)$ is situated in the western Ďumbier part of the Nízke Tatry Mts., $7 \mathrm{~km}$ north from the village Jasenie. The deposit consists of several old mining waste dumps, but orchids occurred only in the heap of the Emil adit, which is located at an altitude of $834 \mathrm{~m}$ a. s.l. E. atrorubens occurs mainly on a sharp slope of a mining heap with almost no moss or lower plants. Few orchids (E. atrorubens and P. bifolia) grew on the horizontal part of the waste dump under trees (especially spruce, willow and birch) or above heap material covered with moss, lower vegetation, and trees. E. atrorubens was extremely widespread in the mining heap and more than 1000 individuals grew on an area about $500 \mathrm{~m}^{2}$. Technogenic substrate is formed mainly of metamorphic rocks of the crystalline basement of the Tatric unit of the Western Carpathians (mostly orthogneisses and migmatites) with fragments of mineralized rocks and also partially ore veins. Parts of ore veins and hydrothermally altered rocks formed hydrothermal carbonates (siderite, dolomite, ankerite, calcite), quartz and baryte and the sulphides are represented mainly as tetrahedrite, galena, bournonite, pyrite, chalcopyrite and sphalerite [20-22]. At the sampling site of the orchid and the substrate, the size of the rock fragments was up to $\sim 7 \mathrm{~cm}$. E. atrorubens grew in a bright habitat, without the presence of other higher plants, mosses and lichens. The slope of the mining heap with orchids was oriented to the south (SSW).

The Ni-Co deposit Dobrá nádej in Dobšiná is located in the Spišsko-Gemerské Rudohorie Mts., in the central part of the Slovak Ore Mountains Mts. in the Inner Western Carpathians ( $48^{\circ} 84^{\prime} 31^{\prime \prime}$, $\left.\mathrm{E} 20^{\circ} 39^{\prime} 08^{\prime \prime}\right)$. The mining heap is situated at an altitude of $807 \mathrm{~m}$ a.s.l. Orchids occur on the vertical part of a heap without vegetation but also in the horizontal part of a waste dump between low spruces and sparse low vegetation represented mainly by grasses. Samples of the orchid E. atrorubens and technogenic substrate were taken from the mining heap, where metamorphic rocks (phyllites, acidic metavolcanites and metasediments) dominate the Gemer superunit. Hydrothermal minerals were represented by siderite, ankerite, dolomite, quartz, hematite, Fe-Ni-Co sulphides, pyrite, chalcopyrite and minerals of tetrahedrite group [20]. Up to $4 \mathrm{~cm}$ was the size of the rock fragments at the sampling point of the plant and substrate. The lighting and botanical conditions at the site and the slope orientation of the mining heap were the same as in the locality Jasenie.

The Fe deposit Sirk-Železník (N 48 $61^{\prime} 71^{\prime \prime}$, E $20^{\circ} 10^{\prime} 54^{\prime \prime}$ ) represents a weathered zone of hydrothermal Fe deposit which is located in the Revúcka vrchovina Highlands, $10 \mathrm{~km}$ west of Jelšava, near the village Sirk at an altitude $416 \mathrm{~m}$ a.s.l. Phyllites, limestones, metaryolite tuffites and metalalydites [23] occurred mostly at this deposit site. Orchids collected for research were found in the forest of a technogenic substrate with a frequent abundance of fungi and incomplete decomposed 
plant residues. This initial substrate was located between pieces of limonite-quartz iron ore $\sim 8-30 \mathrm{~cm}$ in size. No hydrothermal carbonates were present here. Orchids grew in a light deciduous to mixed forest, mostly on a horizontal part of a mining heap between moss-covered pieces of heap material. All individuals of E. pontica grew in one place, in an area of $\sim 15 \mathrm{~m}^{2}$. C. longifolia grew in a disseminated forest a few meters to tens of meters from the occurrence of $E$. pontica.

Further information about localities is given in Račko et al. (2020) [20].

\subsection{Sampling and DNA Extraction}

The samples of the family Orchidaceae were taken from their place of growth. After the plant was collected, the surrounding soil was taken from this area (up to $30 \mathrm{~cm}$ in depth). The roots were washed with distilled water to remove remaining soil particles followed by sterilization, according to Cao et al. (2004) [24] with some modifications, with $70 \%$ ethanol for $5 \mathrm{~min}$, sodium hypochlorite $(2.5 \%$ available chlorine) for $5 \mathrm{~min}$, and again with $70 \%$ ethanol for $1 \mathrm{~min}$. After that, the roots were washed multiple times with distilled water to remove sterilization agents. Surface-sterilized roots were cut into 1-2 $\mathrm{cm}$ pieces and crushed on powder using mortar, pestle, and liquid nitrogen. Isolate II Plant DNA kit (Bioline, Toronto, ON, Canada) was used to extract DNA from these crushed roots. For DNA extraction from soil DNeasy PowerSoil kit (Qiagen, Hilden, Germany) was used.

\subsection{Chemical Analysis}

The $\mathrm{pH}$ of the soil was determined in the distilled $\mathrm{H}_{2} \mathrm{O}$ and $\mathrm{NH}_{4} \mathrm{NO}_{3}$. Oxidisable organic carbon $\left(\% \mathrm{CO}_{\mathrm{x}}\right)$ was analyzed by oxidimetry under laboratory conditions. Collected soils contained various heavy metals and potentially toxic elements above limit values as described in detail at Račko et al. (2020) [20].

\subsection{Polymerase Chain Reaction and Library Preparation for Next-Generation Sequencing}

Bacterial 16S rRNA gene and fungal ITS region were amplified using primer pair sequences for the V3 and V4 region [25] and ITS region [26]. We used 16S Amplicon PCR Forward Primer (5'-TCG TCG GCA GCG TCA GAT GTG TAT AAG AGA CAG CCT ACG GGN GGC WGC AG-3'), $16 \mathrm{~S}$ Amplicon PCR Reverse Primer (5'-GTC TCG TGG GCT CGG AGA TGT GTA TAA GAG ACA GGA CTA CHV GGG TAT CTA ATC C-3'), ITS1 (5'-TCG TCG GCA GCG TCA GAT GTG TAT AAG AGA CAG CTT GGT CAT TTA GAG GAA GTA A-3') and ITS4 (5'-GTC TCG TGG GCT CGG AGA TGT GTA TAA GAG ACA GTC CTC CGC TTA TTG ATA TGC-3'). All primers contained Illumina adapter regions. PCR mixture contained HotStartTaq ${ }^{\circledR}$ Plus Master Mix (Qiagen, Hilden, Germany) $(10 \mu \mathrm{l})$, template DNA (20 ng), and amplicon primers $(2 \mu \mathrm{L}, 1 \mu \mathrm{M})$ in the total reaction volume of $20 \mu \mathrm{l}$. Reaction conditions consisted of an initial $95^{\circ} \mathrm{C}, 3 \mathrm{~min} ; 35$ cycles of $95^{\circ} \mathrm{C}$ for $60 \mathrm{~s}, 60^{\circ} \mathrm{C}, 45 \mathrm{~s}$, and $72{ }^{\circ} \mathrm{C}, 60 \mathrm{~s}$, and final extension of $72{ }^{\circ} \mathrm{C}, 7 \mathrm{~min}$. After Amplicon PCR, samples were purified with DNA Clean \& Concentrator ${ }^{\mathrm{TM}}-25$ (Zymo Research, Irvine, CA, USA).

We used the 16S Metagenomic Sequencing Library Preparation protocol (Illumina Inc., San Diego, CA, USA) to create libraries. Index PCR was performed following the manufacturer's instructions using 2x KAPA HiFi HotStart ReadyMix ( $25 \mu \mathrm{L}$, Kapa Biosystems, Wilmington, MA, USA), Nextera XT Index Primers ( $5 \mu \mathrm{L}$ per sample, Illumina Inc., San Diego, CA, USA), PCR grade water, and $5 \mu \mathrm{L}$ of template DNA/cDNA solution in the total reaction volume of $50 \mu \mathrm{L}$. PCR Clean-Up 2 was performed as described in the protocol, followed by library validation using Qubit dsDNA high sensitivity assay (LifeTechnologies, Eugene, OR, USA) and Agilent ${ }^{\circledR}$ HS DNA Chip (Agilent ${ }^{\circledR}$ Technologies 2100 Bioanalyser, Santa Clara, CA, USA). Finally, DNA libraries were normalized to $4 \mathrm{nM}$ concentration, denatured and processed to the sequencing run on MiSeq instrument (Illumina Inc., San Diego, CA, USA) using a MiSeq Reagent kit v3 (Illumina Inc., San Diego, CA, USA) with the paired-end setting of $2 \times 300$ bp reads. 


\subsection{Data Analysis}

Adapters and low-quality ends of sequenced reads were removed using Trimmomatic (version v0.36) [27], based on quality control statistics generated by FastQC (v0.11.5) [28]. After trimming, fragments without sufficient length of both reads $(>35 \mathrm{bp}$ ) were removed from the data set. All trimmed paired reads with sequence overlaps were merged using PEAR (v0.9.10) [29] and passed to QIIME 2 (v2019.10.0) for microbial analyses [30]. There, fastq files were dereplicated using built-in tool vsearch. Then, chimeras and "borderline chimeras" [31] were removed from the dataset using de novo chimera filtering with default parameters [30]. Operational taxonomic units (OTUs) with $99 \%$ similarity were created with de novo clustering of features.

Taxonomy of OTUs was assigned by QIIME's pre-fitted sklearn-based taxonomy classifier [32]. The pre-fitted classifier was trained by the Naive-Bayes classifier with default parameters on Silva 16S database (Silva release 132) [33] and ITS database (UNITE 8.2) [34]. Only taxons with $<0.1 \%$ of all reads per sample were used for bacteria and $<0.01 \%$ for fungi. Finally, Faith's phylogenetic diversity for all samples was calculated using faith-pd plugin [35]. All computational analyses were written and executed using the SnakeLines framework (v0.9.2) [36,37].

\section{Results and Discussion}

Orchidaceae is a family of rare plants and their presence in metal-rich biotopes has ecological and conservation importance [38]. Orchids colonizing mining heaps rely on the fungal mycelium to obtain the necessary nutrients since their root system is not well developed. They belong to metal excluders, and mycorrhizal fungi seem to play an important role in filtering out the heavy metals [38-40]. Despite these extreme conditions, the environment is sufficiently tolerable to the population of orchids, soil filamentous fungi and bacteria.

In orchidaceous mycorrhiza, fungi have the ability to support the composition of plants in stressed ecological niches and to increase the plant's condition [41]. The properties of orchidaceous mycorrhiza in mycoheterotrophic plants that allow colonization under stress conditions are changes in root morphology, orchid metabolism, such as enzymatic activities, secretion of organic acids with low molecular weight and phenols, and associations with orchideous mycorrhizal fungus adapted to the environmental conditions in which orchids occur $[42,43]$. To identify fungal and bacterial communities in the roots of orchids and surrounding soil in heavy metal contaminated areas we used the marker gene sequencing approach which has been the most commonly used method to analyze these communities. It is simple, fast, cost-effective, and well-tested approach $[44,45]$ in comparison to whole-genome sequencing $[45,46]$.

A total of 171 species of fungi were detected from five samples of orchids, belonging to seven phyla, Ascomycota, Basidiobolomycota, Basidiomycota, Chytridiomycota, Monoblepharomycota, Mortierellomycota, and Mucoromycota (Table 1). The highest biodiversity of fungi was situated in the roots of E. pontica (Sirk), and the surrounding soil of E. atrorubens (Jasenie). Moreover, the most abundant phylum of fungi was Basidiomycota in both soil and the roots of orchids. Furthermore, the most abundant species of fungi in the roots of orchids was Cortinarius saturatus, Mycena sp., Mycena citrinomarginata, Mycenella bryophila, Colletotrichum capsici, Trichophaea pseudogregaria, Phacidium sp., Russula solaris, Mortierella sp., Amphinema byssoides, Mycena sanguinolenta, Amphinema sp., and Piloderma byssinum (Figure 2). Further, in the surrounding soil, it was Cortinarius saturatus, Cortinarius scandens, Helvellosebacina concrescens, Mortierella sp., Cortinarius sp., Helvellosebacina sp., Cuphophyllus virgineus, Tetracladium sp., Colletotrichum capsici, Cortinarius vernus, Penicillium sp., Saitozyma podzolica, and Solicoccozyma terricola (Figure 3). In addition, we observed that the biodiversity in sampled pairs were consistently higher in soil samples, both in the number of discovered species (median 2.7x higher) and the evenness (median 2.66x higher of Faith's phylogenetic diversity). Decreased diversity could be due to the specific environment in the roots of the orchids. 
Table 1. DNA identification of the fungi isolated from the roots of the orchids and surrounding soils.

\begin{tabular}{|c|c|c|c|c|c|c|c|c|c|c|}
\hline \multirow{5}{*}{$\begin{array}{l}\text { The Most Abundant Strains of } \\
\text { Fungi Detected in the Roots of } \\
\text { Orchids and Surrounding Soils }\end{array}$} & \multicolumn{2}{|c|}{1} & \multicolumn{2}{|c|}{2} & \multicolumn{2}{|c|}{3} & \multicolumn{2}{|c|}{4} & \multicolumn{2}{|c|}{5} \\
\hline & \multicolumn{2}{|c|}{$\begin{array}{c}\text { Epipactis } \\
\text { atrorubens }\end{array}$} & \multicolumn{2}{|c|}{$\begin{array}{l}\text { Epipactis } \\
\text { atrorubens }\end{array}$} & $\begin{array}{r}\text { Plato } \\
\text { bif }\end{array}$ & tera & & & $\begin{array}{l}\text { Cepha } \\
\text { lon }\end{array}$ & thera \\
\hline & $\begin{array}{l}\mathrm{Ni-Co} \\
\text { Heap }\end{array}$ & $\begin{array}{l}\text { ining } \\
\text { bšiná }\end{array}$ & $\mathrm{Pb}-\mathrm{i}$ & Minin & Heap Ja & & & Minin & Heap Si & \\
\hline & & & $\mathrm{pH}$ & & & & & & & \\
\hline & Roots & Soil & Roots & Soil & Roots & Soil & Roots & Soil & Roots & Soil \\
\hline Ascomycota & & & & & & & & & & \\
\hline Alatospora acuminata & - & - & - & + & - & - & - & - & - & - \\
\hline Alatospora sp. & - & - & - & + & - & - & - & - & - & - \\
\hline Arthrobotrys conoides & - & + & - & - & - & - & - & - & - & - \\
\hline Arthrobotrys sp. & - & + & - & - & - & - & - & - & - & - \\
\hline Aureobasidium pullulans & - & + & - & - & - & - & - & - & - & - \\
\hline Bradymyces alpinus & - & + & - & - & - & - & - & - & - & - \\
\hline Cadophora sp. & - & + & - & - & - & - & - & - & - & - \\
\hline Capronia sp. & - & - & - & - & - & + & - & - & - & - \\
\hline Cenocoscum geophilum & - & - & - & - & - & - & - & - & + & + \\
\hline Cenococcum sp. & - & - & - & - & - & - & + & + & + & + \\
\hline Cistella sp. & - & - & - & + & - & - & - & - & - & - \\
\hline Cladophialophora minutissima & - & - & - & + & - & - & - & - & - & - \\
\hline Cladosporium sp. & - & + & - & - & - & - & - & - & - & - \\
\hline Colletotrichum capsici & - & + & + & + & + & + & + & + & + & + \\
\hline Dothidea eucalypti & - & - & - & + & - & - & - & - & - & - \\
\hline Exophiala equina & - & - & - & + & - & - & + & + & - & + \\
\hline Exophiala radicis & - & - & - & + & - & - & + & + & - & + \\
\hline Exophiala sp. & - & - & + & + & - & - & + & + & - & + \\
\hline Geopora arenicola & - & + & - & - & - & - & - & - & - & - \\
\hline Geopora sp. & - & + & - & - & - & - & - & - & - & - \\
\hline Geoglossum fallax & - & - & - & - & - & - & + & + & - & - \\
\hline Geomyces auratus & - & - & - & - & - & + & - & - & - & - \\
\hline Gyoerffyella sp. & - & - & - & - & - & - & + & - & - & - \\
\hline Herpotrichia sp. & - & - & - & - & - & - & - & - & + & - \\
\hline Humaria hemisphaerica & - & - & - & - & - & - & + & - & - & - \\
\hline Humaria sp. & - & - & - & - & - & - & + & - & - & - \\
\hline Hyalodendriella_betulae & - & - & - & + & - & - & - & - & - & - \\
\hline Hyalodendriella sp. & - & - & - & + & - & - & - & - & - & - \\
\hline Hyaloscypha bicolor & - & - & - & - & - & + & - & + & - & - \\
\hline Hyaloscypha finlandica & - & - & - & + & - & - & - & + & + & - \\
\hline Hyaloscypha sp. & - & - & - & - & - & - & - & - & + & - \\
\hline Infundichalara minuta & - & - & - & + & - & - & - & - & - & - \\
\hline Lachnum sp. & - & - & - & - & - & - & + & - & - & - \\
\hline Leohumicola sp. & - & - & - & + & - & - & - & - & - & - \\
\hline Mollisia dextrinospora & - & - & - & + & - & - & - & - & - & - \\
\hline Sporormiella intermedia & - & - & - & + & - & - & - & - & - & - \\
\hline Paracladophialophora carceris & - & - & - & - & - & - & - & + & - & - \\
\hline Paraphoma fimeti & - & + & - & - & - & - & - & - & - & - \\
\hline Penicillium brunneoconidiatum & - & - & - & + & - & + & - & - & - & - \\
\hline Penicillium spinulosum & - & - & - & + & - & + & - & - & - & - \\
\hline Penicillium sp. & - & - & - & + & - & + & - & + & - & + \\
\hline Peziza succosa & - & - & - & + & - & - & - & + & - & - \\
\hline Pezoloma ericae & - & - & + & + & - & + & - & + & + & - \\
\hline Phacidium sp. & - & - & + & - & - & - & - & - & - & - \\
\hline Phialocephala fortinii & - & - & - & - & + & - & - & - & - & - \\
\hline Phialocephala sp. & - & - & - & - & - & + & - & - & - & - \\
\hline Plenodomus biglobosus & - & + & - & - & - & - & - & - & - & - \\
\hline Pseudodictyosporium elegans & - & + & - & - & - & - & - & - & - & - \\
\hline Pseudodictyosporium sp. & - & + & - & - & - & - & - & - & - & - \\
\hline Sagenomella sp. & - & - & - & - & - & + & - & - & - & - \\
\hline Talaromyces sp. & - & - & - & + & - & + & - & - & - & - \\
\hline Tetracladium apiense & - & - & + & - & - & - & - & - & - & - \\
\hline Tetracladium sp. & - & + & + & + & - & - & - & - & - & - \\
\hline Tricharina gilva & - & + & - & - & - & - & - & - & - & - \\
\hline Tricharina sp. & - & + & - & - & - & - & - & - & - & - \\
\hline Trichocladium opacum & - & - & - & - & - & - & - & + & - & - \\
\hline Trichophaea pseudogregaria & + & + & - & - & - & - & - & - & - & - \\
\hline Trichophaea woolhopeia & + & - & + & + & - & - & - & - & - & - \\
\hline Tuber rufum & - & - & - & - & - & - & - & - & - & + \\
\hline Tuber sp. & - & - & - & - & - & - & - & - & - & + \\
\hline Verrucaria muralis & - & + & - & - & - & - & - & - & - & - \\
\hline Verrucaria ahtii & - & + & - & - & - & - & - & - & - & - \\
\hline Basidiobolomycota & & & & & & & & & & \\
\hline Basidiobolus magnus & - & - & - & + & - & - & - & - & - & - \\
\hline Basidiobolus ranarum & - & - & - & + & - & - & - & - & - & - \\
\hline
\end{tabular}


Table 1. Cont.

\begin{tabular}{|c|c|c|c|c|c|c|c|c|c|c|}
\hline \multirow{5}{*}{$\begin{array}{l}\text { The Most Abundant Strains of } \\
\text { Fungi Detected in the Roots of } \\
\text { Orchids and Surrounding Soils }\end{array}$} & \multicolumn{2}{|c|}{1} & \multicolumn{2}{|c|}{2} & \multicolumn{2}{|c|}{3} & \multicolumn{2}{|c|}{4} & \multicolumn{2}{|c|}{5} \\
\hline & \multicolumn{2}{|c|}{$\begin{array}{l}\text { Epipactis } \\
\text { atrorubens }\end{array}$} & \multicolumn{2}{|c|}{$\begin{array}{l}\text { Epipactis } \\
\text { atrorubens }\end{array}$} & $\begin{array}{r}\text { Plato } \\
\text { bif }\end{array}$ & $\begin{array}{l}\text { hera } \\
\text { ia }\end{array}$ & & & $\begin{array}{r}\text { Cepha } \\
\text { lon }\end{array}$ & $\begin{array}{l}\text { thera } \\
\text { lia }\end{array}$ \\
\hline & $\begin{array}{l}\text { Ni-Co } \\
\text { Heap }\end{array}$ & $\begin{array}{l}\text { ining } \\
\text { bšiná }\end{array}$ & & Mini & Heap Ja & & & Mini & Heap Si & \\
\hline & & & & & & & & & & \\
\hline & Roots & Soil & Roots & Soil & Roots & Soil & Roots & Soil & Roots & Soil \\
\hline Basidiomycota & & & & & & & & & & \\
\hline Amphinema byssoides & - & - & + & + & - & - & - & - & - & - \\
\hline Amphinema sp. & - & - & + & + & - & + & - & - & - & - \\
\hline Apiotrichum dulcitum & - & - & - & - & - & - & - & + & - & - \\
\hline Apiotrichum sp. & - & - & - & - & - & - & - & + & - & - \\
\hline Coprinellus micaceus & - & - & - & + & - & - & - & - & - & - \\
\hline Cortinarius casimiri & - & - & - & - & - & + & - & - & - & - \\
\hline Cortinarius cyprinus & - & - & - & - & - & - & - & - & - & + \\
\hline Cortinarius saturatus & + & + & + & + & + & + & + & + & + & + \\
\hline Cortinarius scandens & - & - & - & - & + & + & - & - & - & - \\
\hline Cortinarius subtilis & - & - & - & + & - & - & - & - & - & - \\
\hline Cortinarius vernus & - & - & - & - & - & + & + & + & - & - \\
\hline Cortinarius sp. & - & - & - & + & + & + & + & + & - & + \\
\hline Cuphophyllus virgineus & - & - & - & - & - & - & + & + & - & - \\
\hline Cuphophyllus sp. & - & - & - & - & - & - & - & + & - & - \\
\hline Cutaneotrichosporon moniliiforme & - & - & - & - & - & - & - & - & + & - \\
\hline Cutaneotrichosporon sp. & - & - & - & - & - & - & + & - & - & - \\
\hline Filobasidium wieringae & - & + & - & - & - & - & - & - & - & - \\
\hline Flagelloscypha minutissima & - & - & - & - & - & - & - & - & - & + \\
\hline Ganoderma sp. & - & - & - & + & - & - & - & + & - & + \\
\hline Hebeloma cylindrosporum & - & - & + & - & - & - & - & - & - & - \\
\hline Hebeloma leucosarx & - & - & + & - & - & - & - & - & - & - \\
\hline Hebeloma sp. & - & - & + & + & - & - & - & - & - & - \\
\hline Helvellosebacina concrescens & - & - & - & - & - & - & - & - & + & + \\
\hline Helvellosebacina sp. & - & - & - & - & - & - & + & + & - & + \\
\hline Hymenogaster griseus & - & - & - & - & - & - & - & + & - & + \\
\hline Hymenogaster rehsteineri & - & - & - & - & - & - & - & - & - & + \\
\hline Hymenogaster sp. & - & - & - & - & - & - & - & - & - & + \\
\hline Hypholoma fasciculare & - & - & - & + & - & - & - & - & - & - \\
\hline Inocybe mixtilis & - & - & - & - & - & - & - & - & - & + \\
\hline Inocybe sp. & - & + & - & - & - & - & - & - & - & - \\
\hline Laccaria sp. & - & - & - & - & - & - & - & + & - & - \\
\hline Lactarius circellatus & - & - & - & - & - & - & - & + & - & - \\
\hline Lactarius torminosus & - & - & - & - & + & - & - & - & - & - \\
\hline Lactarius sp. & - & - & - & - & + & - & - & - & - & - \\
\hline Leccinum pseudoscabrum & - & - & - & - & - & - & + & + & - & - \\
\hline Lycoperdon pyriforme & - & - & - & + & - & - & - & - & - & - \\
\hline Lycoperdon sp. & - & - & - & + & - & - & - & - & - & - \\
\hline Macrolepiota procera & - & - & - & - & - & - & - & + & - & - \\
\hline Mallocybe sp. & - & + & - & - & - & - & - & - & - & - \\
\hline Mycena citrinomarginata & - & - & - & - & - & - & + & + & + & - \\
\hline Mycena leptocephala & - & - & - & - & - & - & + & + & - & - \\
\hline Mycena olivaceomarginata & - & - & - & - & - & - & + & - & + & - \\
\hline Mycena plumipes & - & - & - & - & - & - & + & + & - & - \\
\hline Mycena pura & - & - & - & - & - & + & - & - & - & - \\
\hline Mycena sanguinolenta & - & - & + & + & - & - & - & - & - & - \\
\hline Mycena vulgaris & - & - & - & - & + & + & - & - & - & - \\
\hline Mycena sp. & - & - & + & - & + & + & + & + & + & + \\
\hline Mycenella bryophila & - & - & - & - & - & - & - & - & + & - \\
\hline Mycenella trachyspora & - & - & - & - & - & - & - & - & + & - \\
\hline Mycenella sp. & - & - & - & - & - & - & - & - & + & - \\
\hline Odontia fibrosa & - & - & - & + & - & - & - & - & - & - \\
\hline Odontia sp. & - & - & - & + & - & - & - & - & - & - \\
\hline Phallus impudicus & - & - & - & + & - & - & - & + & - & + \\
\hline Phallus ultraduplicatus & - & - & - & + & - & - & - & - & - & - \\
\hline Phallus sp. & - & - & - & + & - & - & - & - & - & - \\
\hline Piloderma byssinum & + & - & - & + & - & - & + & + & - & - \\
\hline Psathyrella candolleana & - & - & - & - & - & - & - & - & - & + \\
\hline Psathyrella sp. & - & - & - & - & - & - & - & - & - & + \\
\hline Russula persicina & - & - & - & - & - & - & - & - & + & - \\
\hline Russula solaris & - & - & - & - & - & - & + & + & + & + \\
\hline Russula versicolor & - & - & - & - & - & - & + & - & - & - \\
\hline Russula sp. & - & - & - & - & - & - & + & + & + & + \\
\hline Saitozyma podzolica & - & - & - & + & - & - & + & + & - & + \\
\hline Saitozyma sp. & - & - & - & - & - & - & - & + & - & + \\
\hline Scleroderma bovista & - & - & - & - & - & - & - & - & - & + \\
\hline Scleroderma sp. & - & - & - & - & - & - & - & - & - & + \\
\hline
\end{tabular}


Table 1. Cont.

\begin{tabular}{|c|c|c|c|c|c|c|c|c|c|c|}
\hline \multirow{5}{*}{$\begin{array}{l}\text { The Most Abundant Strains of } \\
\text { Fungi Detected in the Roots of } \\
\text { Orchids and Surrounding Soils }\end{array}$} & \multicolumn{2}{|c|}{1} & \multicolumn{2}{|c|}{2} & \multicolumn{2}{|c|}{3} & \multicolumn{2}{|c|}{4} & \multicolumn{2}{|c|}{5} \\
\hline & \multicolumn{2}{|c|}{$\begin{array}{l}\text { Epipactis } \\
\text { atrorubens }\end{array}$} & \multicolumn{2}{|c|}{$\begin{array}{c}\text { Epipactis } \\
\text { atrorubens }\end{array}$} & \multicolumn{2}{|c|}{$\begin{array}{l}\text { Platanthera } \\
\text { bifolia }\end{array}$} & \multicolumn{2}{|c|}{$\begin{array}{l}\text { Epipactis } \\
\text { pontica }\end{array}$} & \multicolumn{2}{|c|}{$\begin{array}{l}\text { Cephalanthera } \\
\text { longifolia }\end{array}$} \\
\hline & \multicolumn{2}{|c|}{$\begin{array}{l}\text { Ni-Co Mining } \\
\text { Heap Dobšiná }\end{array}$} & \multicolumn{4}{|c|}{$\mathrm{Pb}-\mathrm{Zn}$ Mining Heap Jasenie } & \multicolumn{4}{|c|}{ Fe Mining Heap Sirk } \\
\hline & \multicolumn{2}{|c|}{ pH 7.73} & \multicolumn{2}{|c|}{ pH 7.36} & \multicolumn{2}{|c|}{ pH 7.81} & \multicolumn{2}{|c|}{ pH 4.98} & & \\
\hline & Roots & Soil & Roots & Soil & Roots & Soil & Roots & Soil & Roots & Soil \\
\hline Sebacina sp. & - & - & - & - & - & - & + & + & - & - \\
\hline Solicoccozyma terricola & - & - & - & + & - & - & + & + & - & + \\
\hline Solicoccozyma sp. & - & - & - & - & - & - & - & + & - & - \\
\hline Tephrocybe rancida & - & - & - & - & - & - & - & + & - & - \\
\hline Thanatephorus sp. & + & - & - & - & - & - & - & - & - & - \\
\hline Thelephora atra & - & - & + & + & - & - & + & + & - & - \\
\hline Thelephora caryophyllea & - & - & - & + & - & - & - & - & - & - \\
\hline Tomentella badia & - & - & - & - & - & - & + & + & - & - \\
\hline Tomentella fuscocinerea & - & - & + & - & - & - & - & - & - & - \\
\hline Tomentella lilacinogrisea & - & - & - & + & - & - & - & - & - & - \\
\hline Tomentella pilosa & - & - & - & - & - & - & + & + & - & + \\
\hline Tomentella sp. & + & + & - & + & - & + & + & + & - & + \\
\hline Tremella sp. & - & - & - & - & - & - & - & - & - & + \\
\hline Tricholoma argyraceum & - & - & + & + & - & - & - & - & - & - \\
\hline Tricholoma sp. & - & - & + & + & - & - & - & - & - & - \\
\hline Tylospora sp. & - & + & + & - & - & - & - & - & - & - \\
\hline Chytridiomycota & & & & & & & & & & \\
\hline Operculomyces laminatus & - & - & - & + & - & - & - & - & - & - \\
\hline Rhizophlyctis rosea & - & - & - & + & - & - & - & - & - & - \\
\hline Rhizophydium sp. & - & + & - & - & - & - & - & - & - & - \\
\hline Spizellomyces pseudodichotomus & - & + & - & - & - & - & - & - & - & - \\
\hline Monoblepharomycota & & & & & & & & & & \\
\hline Sanchytrium sp. & - & - & - & + & - & - & - & - & - & + \\
\hline Mortierellomycota & & & & & & & & & & \\
\hline Mortierella alpina & - & + & - & - & - & - & - & - & - & + \\
\hline Mortierella amoeboidea & - & - & - & - & - & - & - & - & + & + \\
\hline Mortierella beljakovae & - & - & - & - & - & - & - & - & - & + \\
\hline Mortierella clonocystis & - & - & - & + & - & - & - & + & - & - \\
\hline Mortierella gamsii & - & - & - & - & - & - & + & + & - & + \\
\hline Mortierella globulifera & - & - & - & - & - & - & - & + & - & - \\
\hline Mortierella humilis & - & - & - & + & - & + & - & + & - & - \\
\hline Mortierella minutissima & - & - & - & + & - & - & + & + & - & - \\
\hline Mortierella paraensis & - & - & - & - & - & - & - & + & - & - \\
\hline Mortierella pseudozygospora & - & - & - & - & - & - & - & + & - & + \\
\hline Mortierella sarnyensis & - & - & - & - & - & - & - & + & - & + \\
\hline Mortierella zonata & - & - & - & - & - & - & - & + & - & + \\
\hline Mortierella sp. & - & + & + & + & - & + & + & + & + & + \\
\hline Mucoromycota & & & & & & & & & & \\
\hline Absidia cylindrospora & - & - & - & - & - & - & - & - & - & + \\
\hline Absidia sp. & - & - & - & - & - & - & - & + & - & - \\
\hline Mucor hiemalis & - & - & - & - & - & - & - & + & - & - \\
\hline Mucor sp. & - & - & - & + & - & - & - & - & - & - \\
\hline Umbelopsis isabellina & - & - & - & - & - & + & - & - & - & - \\
\hline Umbelopsis sp. & - & - & - & + & - & + & - & - & - & - \\
\hline Zygorhynchus sp. & - & - & - & + & - & - & - & - & - & - \\
\hline$\Sigma 171$ species & 6 & 29 & 21 & 62 & 9 & 25 & 36 & 57 & 21 & 44 \\
\hline Faith's phylogenetic diversity & 509 & 2828 & 989 & 3378 & 429 & 1141 & 1053 & 2368 & 906 & 2190 \\
\hline
\end{tabular}

The $\mathrm{pH}$ value is a key factor affecting the plant, fungal and bacterial communities on the mining dumps. Soil fungi can grow over a broad range of $\mathrm{pH}$ [47], however, soil bacteria are more sensitive to low $\mathrm{pH}$ [48], so fungi can dominate this $\mathrm{pH}$ spectrum. Values of $\mathrm{pH}$ varied from acidic (4.98) to slightly alkaline (7.81).

A high number (57) of fungal species, found in the surrounding soil, tolerate acidic $\mathrm{pH}$ of 4.98 . Regarding roots, it was 37. Similar to our study, fungi like Cladophialophora sp., Cladosporium sp., Exophiala sp., Mortierella alpina, Mortierella sp., Mucor sp., Mucor hiemalis, Penicillium sp., Umbelopsis sp. provided a great tolerance to a higher concentration of some heavy metals in high acidic to neutral $\mathrm{pH}$ of the soils [49]. In regard to to $\mathrm{pH}$, we did not observe anything significant or different in comparison to other studies [50-52]. Organisms from extreme habitats have developed strategies for 
survival and growth in harsh conditions in the production of organic molecules [53]. They have the ability of adaptation to high salinity, radiation, limited nutrients, extreme temperatures and pressure, and variable values of the acidity [54]. In these contaminated areas, metal-resistant fungi occur which have potential in the process of bioremediation [55].

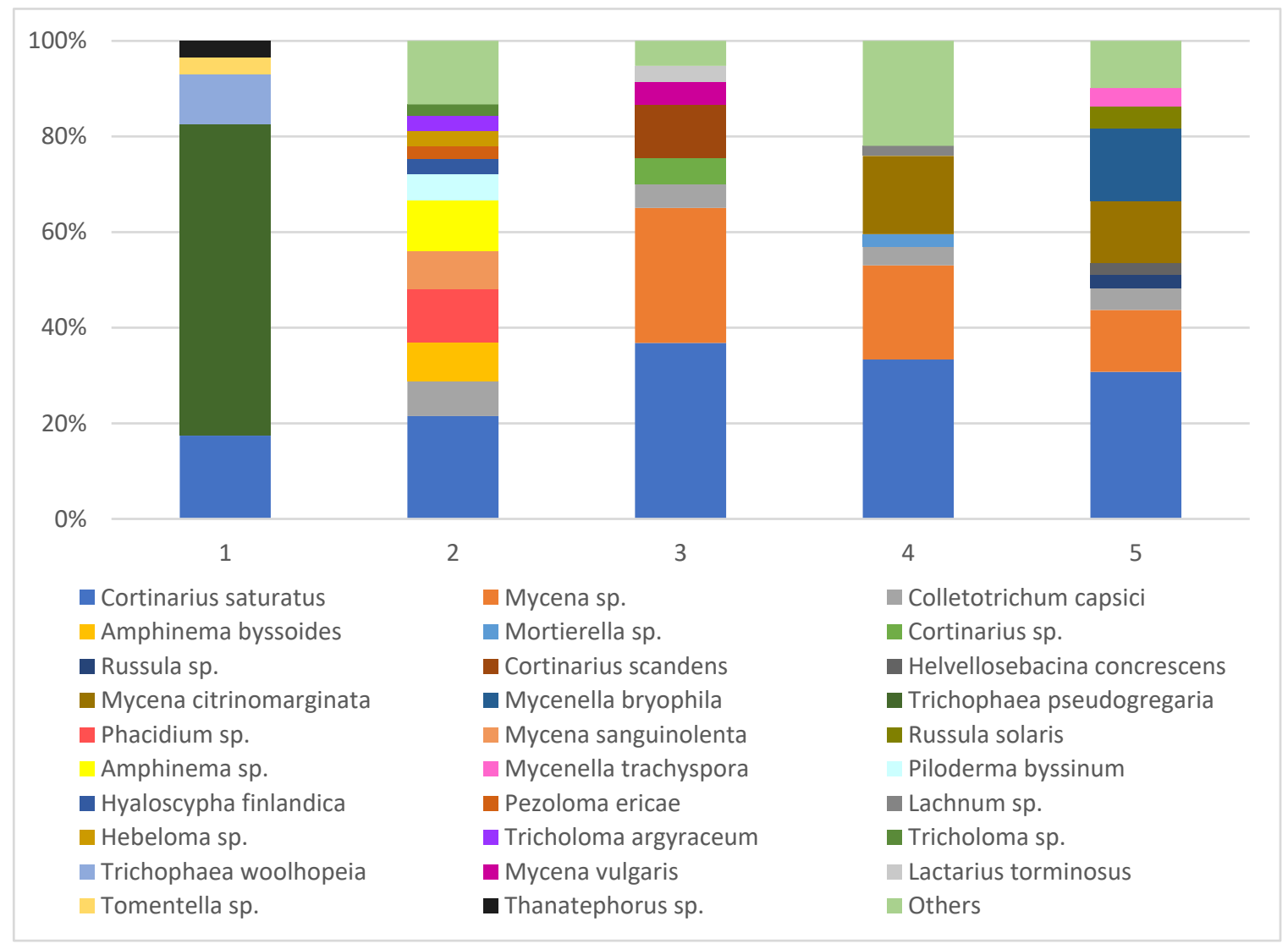

Figure 2. Fungal communities detected in the roots of orchids. (1) E. atrorubens (Dobšiná); (2) E. atrorubens (Jasenie); (3) P. bifolia (Jasenie); (4) E. pontica (Sirk); (5) C. longifolia (Sirk).

The benefits of mycorrhizas to both plants and fungi is an uptake of nutrients. The fungi obtain carbon from the plants, and in return, enhance a plant's ability to take up minerals or water, subsequently leading to improvement of plant growth and reproduction [56]. Many species of fungi possess the ability to act as an effective biosorbent of the toxic metal. However, these elements may inhibit their growth and affect their reproduction [57].

Species, such as Colletotrichum capsici, Cortinarius saturatus, Cortinarius sp., Mortierella sp., Penicillium sp., and Tomentella sp. were present in soil in four or more samples. Regarding roots, it was Mycena sp., Colletotrichum capsici, Cortinarius saturatus.

The composition of fungi in orchid roots is usually much more uniform for orchid species growing in a forest community in the presence of more species of higher plants and trees than for orchids that form plant monocultures on the stony substrate of mining heaps. This difference is not so significant for the fungi present in the soil.

At the same time, the study of fungi in the roots of different species of orchids from different types of habitats and the surrounding technogenic substrate showed that the only species of fungus that occurred in an increased quantity of all the samples was Cortinarius saturatus. In all other fungi, their quantitative composition in the soils and roots of orchids did not correlate. In many cases, significantly present species in the roots were almost non-existent in the soil, and vice versa. 
In the study of fungi from the mining sites [52] and recent study of microscopic fungi from five substrates of old environmental loads [50], species, such as Cladosporium sp., Exophiala sp., Mortierella alpina, Mortierella sp., Mucor hiemalis, Mucor sp., Penicillium spinulosum, Penicillium sp., Phialocephala sp., and Umbelopsis sp. agreed with our results.

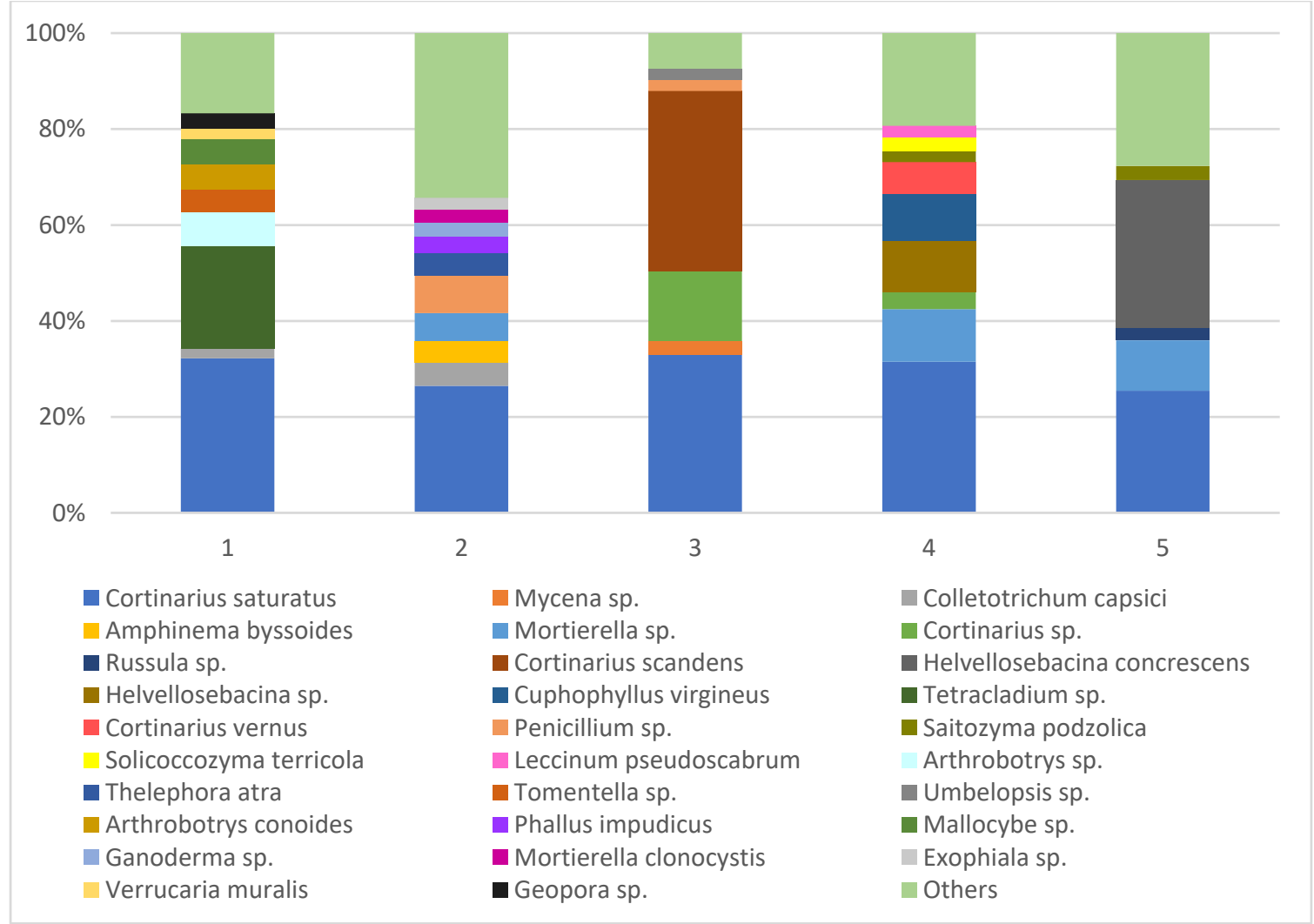

Figure 3. Fungal communities detected in the surrounding soil of orchids. (1) E. atrorubens (Dobšiná); (2) E. atrorubens (Jasenie); (3) P. bifolia (Jasenie); (4) E. pontica (Sirk); (5) C. longifolia (Sirk).

The function of the genus Mycena, which is one of the representatives to be present in four samples, is to influence the growth of roots [58]. Despite the great biodiversity of fungal genera present in the individual samples, a comparison of the root and soil samples suggests the specificity of both for the host plant. In another study by Cui et al. 2017 [59], Mortierella sp. effectively reduced the heavy metals and significantly shortened the remediation period and improved the poor habitat of mine tailings. Bioaccumulation and biosorption abilities of Penicillium spp. were also studied [60-62].

\section{Bacterial Communities}

Bacteria have high importance in industrial processes, bioremediation, and heavy metal tolerance [13]. Bioremediation is the process of biological systems cleaning of the polluted environment by the bacteria and fungi, which are important for reclamation, immobilization and detoxification of heavy metals [63].

A total of 30 species of bacteria were detected from five samples of orchids, belonging to eight phyla, Acidobacteria, Actinobacteria, Bacteroidetes, Gemmatimonadetes, Planctomyces, Proteobacteria, Verrucomicrobia, and Tenericutes (Table 2). The highest biodiversity of bacteria was in the roots and also surrounding soil of E. atrorubens (Jasenie). Additionally, the evenness in sampled pairs was consistently higher in soil samples (median 1.2x higher of Faith's phylogenetic diversity). However, that was not the cause in the number of discovered species as it was in analysis of fungal communities. 
Table 2. DNA identification of the bacteria isolated from the roots of the orchids and surrounding soils.

\begin{tabular}{|c|c|c|c|c|c|c|c|c|c|c|}
\hline \multirow{5}{*}{$\begin{array}{l}\text { The Most Abundant Strains } \\
\text { of Bacteria Detected in the } \\
\text { Roots of Orchids and } \\
\text { Surrounding Soils }\end{array}$} & \multicolumn{2}{|c|}{1} & \multicolumn{2}{|c|}{2} & \multicolumn{2}{|c|}{3} & \multicolumn{2}{|c|}{4} & \multicolumn{2}{|c|}{5} \\
\hline & \multicolumn{2}{|c|}{$\begin{array}{c}\text { Epipactis } \\
\text { atrorubens }\end{array}$} & \multicolumn{2}{|c|}{$\begin{array}{l}\text { Epipactis } \\
\text { atrorubens }\end{array}$} & \multicolumn{2}{|c|}{$\begin{array}{l}\text { Platanthera } \\
\text { bifolia }\end{array}$} & \multicolumn{2}{|c|}{$\begin{array}{l}\text { Epipactis } \\
\text { pontica }\end{array}$} & \multicolumn{2}{|c|}{$\begin{array}{l}\text { Cephalanthera } \\
\text { longifolia }\end{array}$} \\
\hline & \multicolumn{2}{|c|}{$\begin{array}{l}\text { Ni-Co Mining } \\
\text { Heap Dobšiná }\end{array}$} & \multicolumn{4}{|c|}{ Pb-Zn Mining Heap Jasenie } & \multicolumn{4}{|c|}{ Fe Mining Heap Sirk } \\
\hline & \multicolumn{2}{|c|}{ pH 7.73} & \multicolumn{2}{|c|}{ pH 7.36} & \multicolumn{2}{|c|}{ pH 7.81} & \multicolumn{2}{|c|}{ pH 4.98} & \multicolumn{2}{|c|}{ pH 6.58} \\
\hline & Roots & Soil & Roots & Soil & Roots & Soil & Roots & Soil & Roots & Soil \\
\hline \multicolumn{11}{|l|}{ Acidobacteria } \\
\hline Candidatus solibacter & - & - & + & + & - & + & - & + & - & + \\
\hline Pyrinomonadaceae RB41 & - & + & - & - & - & - & - & - & - & - \\
\hline Vicinamibacter sp. & + & - & - & - & - & - & - & - & - & - \\
\hline \multicolumn{11}{|l|}{ Actinobacteria } \\
\hline Nocardioides sp. & + & - & + & + & - & - & + & - & - & - \\
\hline Bacteroidetes & & & & & & & & & & \\
\hline Chryseobacterium sp. & - & - & - & - & - & - & + & - & - & - \\
\hline Flavobacterium sp. & - & - & + & - & - & - & - & - & - & - \\
\hline Pedobacter sp. & - & - & - & - & - & - & + & - & - & - \\
\hline Gemmatimonadetes & & & & & & & & & & \\
\hline Gemmatimonas sp. & - & + & - & - & - & - & - & - & - & - \\
\hline Planctomycetes & & & & & & & & & & \\
\hline Fimbriiglobus sp. & - & - & + & + & - & - & - & - & - & - \\
\hline Gemmata sp. & + & - & + & + & + & - & + & + & + & - \\
\hline Pirellulaceae Pir4 lineage & - & - & + & + & - & - & - & - & - & - \\
\hline Pirellula sp. & + & + & + & + & - & - & - & - & - & + \\
\hline Rubinisphaeraceae SH-PL14 & + & + & + & + & - & - & - & - & - & - \\
\hline Singulisphaera sp. & - & - & - & - & + & + & + & - & + & - \\
\hline Proteobacteria & & & & & & & & & & \\
\hline Acidibacter sp. & - & - & - & - & - & + & - & - & - & - \\
\hline $\begin{array}{l}\text { Allorhizobium-Neorhizobium- } \\
\text { Pararhizobium-Rhizobium }\end{array}$ & - & - & + & - & + & - & + & - & - & - \\
\hline Bradyrhizobium sp. & - & - & + & - & - & + & - & - & - & - \\
\hline $\begin{array}{l}\text { Burkholderia-Caballeronia- } \\
\text { Paraburkholderia }\end{array}$ & - & - & - & - & - & + & + & - & - & - \\
\hline Nitrosomonadaceae Ellin6067 & - & - & - & + & - & - & - & - & - & - \\
\hline Sphingomonas sp. & - & - & + & - & - & - & - & - & - & - \\
\hline Pseudomonas sp. & - & - & - & - & - & - & + & - & - & - \\
\hline Tenericutes & & & & & & & & & & \\
\hline Candidatus phytoplasma fragariae & - & - & - & - & - & - & - & - & + & - \\
\hline Verrucomicrobia & & & & & & & & & & \\
\hline Candidatus udaeobacter & - & + & + & + & - & + & + & + & + & + \\
\hline Candidatus xiphinematobacter & - & - & + & - & - & - & - & + & - & - \\
\hline Chthoniobacter sp. & + & + & + & + & + & - & + & + & + & + \\
\hline Luteolibacter sp. & - & - & + & - & - & _- & - & - & - & - \\
\hline Spartobacteria bacterium WX31 & - & - & - & - & - & - & + & + & + & + \\
\hline AG-212-E04 & - & - & - & - & - & - & - & + & - & - \\
\hline $\begin{array}{l}\text { Verrucomicrobiaceae bacterium } \\
\text { CNC16 }\end{array}$ & + & - & - & - & _- & - & - & - & _- & - \\
\hline Verrucomicrobium sp. & + & - & + & - & - & - & - & - & - & - \\
\hline$\Sigma 30$ species & 8 & 6 & 16 & 10 & 4 & 6 & 11 & 7 & 6 & 5 \\
\hline Faith's phylogenetic diversity & 21.2 & 25.4 & 23.5 & 26.8 & 19.3 & 41.7 & 16.8 & 19.9 & 17.3 & 26.2 \\
\hline
\end{tabular}

Our results show (Figures 4 and 5) that Candidatus Udaeobacter was the dominant bacterium at the Sirk deposit in the roots of two different orchids as well as in technogenic substrate and a high dominance of bacteria from the family Pyrinomonadaceae in a stony substrate on the mining heap in Dobšiná and the related very dominant abundance of the fungus Trichophaea pseudogregaria in the roots of E. atrorubens. The most abundant species in the roots of orchids was Candidatus udaeobacter, Gemmata sp., Singulisphaera sp., Chthoniobacter sp., Pseudomonas sp., Luteolibacter sp., Nocardioides sp., Pirellula sp. (Figure 4). In the surrounding soil, it was Candidatus udaeobacter, Chthoniobacter sp., Pyrinomonadaceae; RB41, Gemmata sp., and Pirellula sp. (Figure 5). Moreover, Candidatus udaeobacter, Candidatus solibacter, and Chthoniobacter sp. were present in four or more soil samples. Furthermore, 11 species tolerated $\mathrm{pH}$ of 4.98 . 


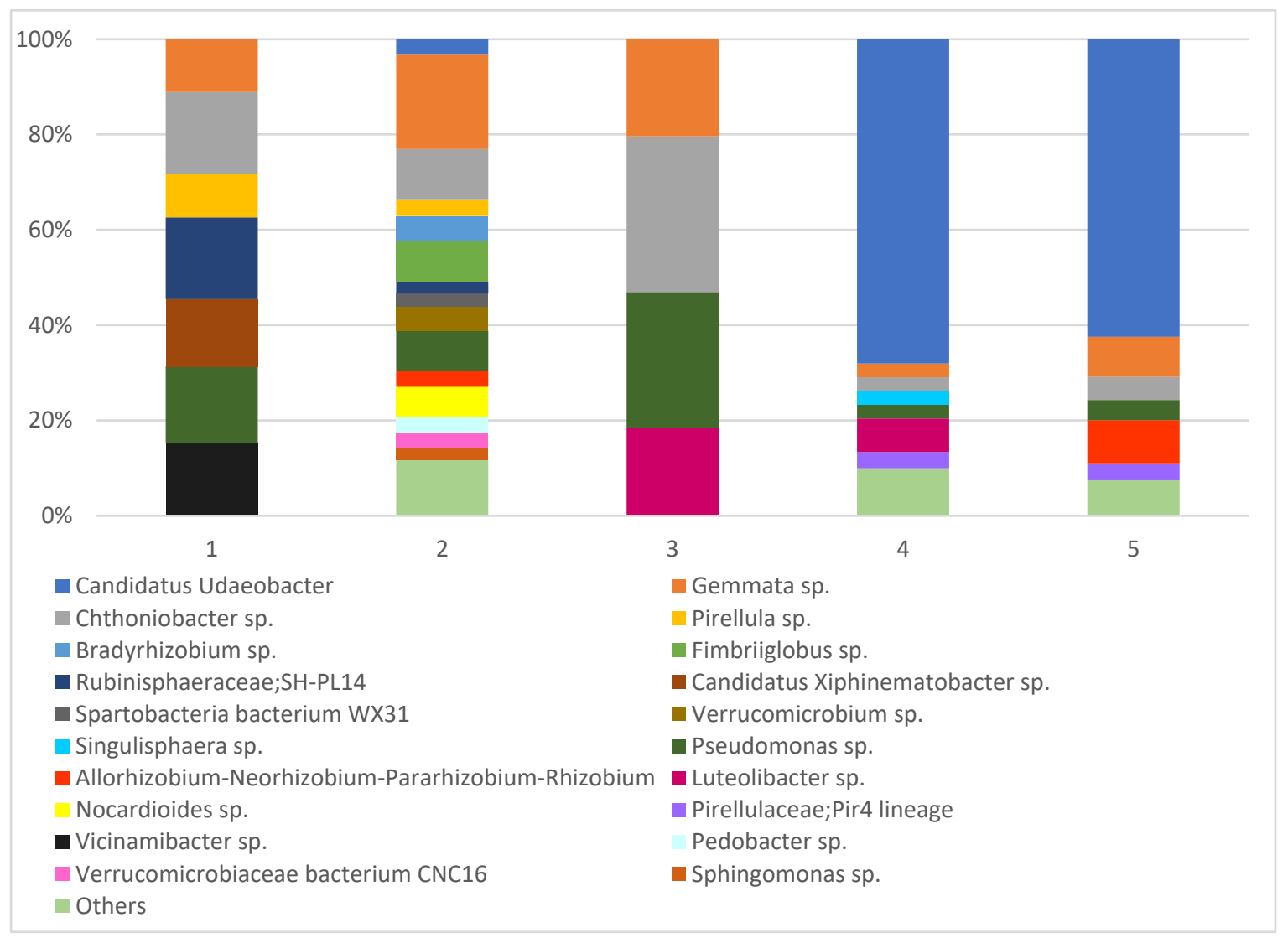

Figure 4. Bacterial communities detected in the roots of orchids. (1) E. atrorubens (Dobšiná); (2) E. atrorubens (Jasenie); (3) P. bifolia (Jasenie); (4) E. pontica (Sirk); (5) C. longifolia (Sirk).

Species, such as Candidatus solibacter, Bradyrhizobium sp., Burkholderia sp., Gemmatimonas sp., Fimbriiglobus sp., Flavobacterium sp., Pseudomonas sp., Sphingomonas sp., Pedobacter sp., and Verrucomicrobia sp. were also detected in heavy metal contaminated soils [64-68]. A bacterium with the highest relative abundance was Candidatus udaeobacter, which is an abundant soil bacterium that reduces its requirement for soil organic carbon by acquiring costly amino acids and vitamins from the environment [69]. Pirellula sp. found in both roots and soil gains energy from the aerobic oxidation of mono- or disaccharides derived from the cleavage of sulfated polymers produced by algae [70].

Bacteria from the genus Pseudomonas induce auxin production by plant growth-promoting bacteria [71]. They are also known as very metabolically active bacteria able to utilize many agrochemicals [72-74]. Together with Luteolibacter sp., Vicinamibacter sp., and Sphingomonas sp. they were identified by many other studies as an oil-degrading bacteria which is important for bioremediation [75-77]. Acidibacter sp. and Candidatus solibacter were suitable to be grown in oil-contaminated soils [78].

The genera representing the heterotrophic strain Planctomycetes have the ability to reduce sulfur and, in particular, play an important role in sugar metabolism [79]. The overlap of the genera present indicates a close connection of the plant with the microbiome, therefore the plant is able to adapt quickly to changing conditions. Nitrosomonadaceae is a strictly aerobic bacteria which has a chemolithoautotrophic metabolism using ammonia as an energy source producing nitrite [80]. Nocardioides spp. are often isolated as plant endophytes and are known to be capable of suppressing crop pathogens [81]. Together with the members of the genus Flavobacterium, they are widely distributed in various environments such as soil, water, and sediment [82].

According to E. atrorubens which was studied at two different localities, we showed that it is not important what species of mycorrhizal fungi and bacteria are associated with orchids, but their 
presence in the substrate. Therefore, the studied orchids can grow in technogenic or anthropogenic soils and substrates as well as other extreme habitats. The species composition of mycorrhizal fungi in the roots of E. atrorubens is very variable in each locality apart from the Cortinarius saturatus species, which is present in all examined roots of orchids. No other species had a significant quantitative representation in both localities of this orchid. Our research has shown that E. atrorubens can use either one dominant fungus (Dobšiná deposit, up to almost 65\% representation of the species Trichophaea pseudogregaria) or even a larger number of species of quantitatively under-represented fungi (Jasenie deposit) for its enormous occurrence in these localities.

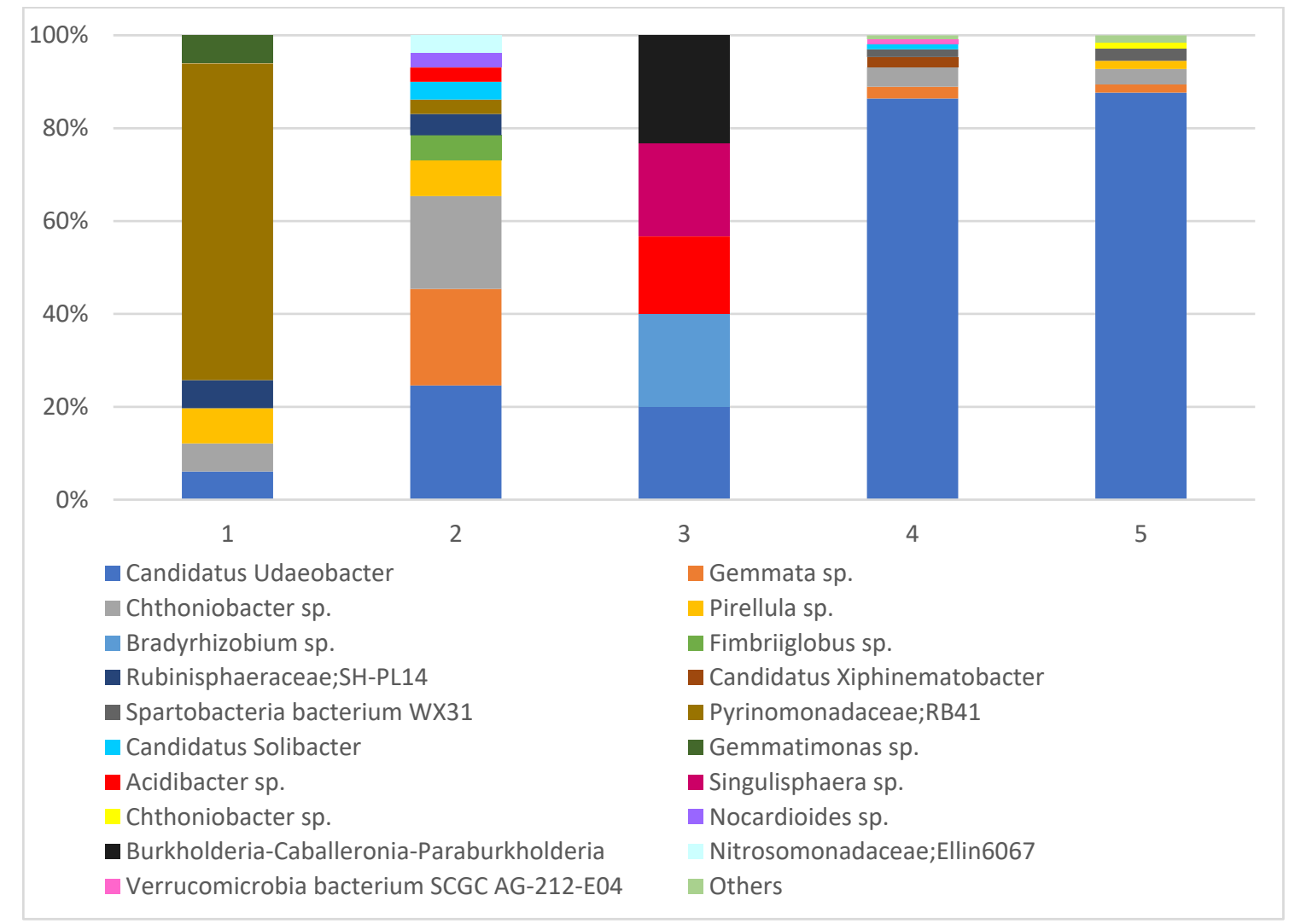

Figure 5. Bacterial communities detected in the surrounding soil of orchids. (1) E. atrorubens (Dobšiná); (2) E. atrorubens (Jasenie); (3) P. bifolia (Jasenie); (4) E. pontica (Sirk); (5) C. longifolia (Sirk).

\section{Conclusions}

Due to its adaptation and survival strategy, the family Orchidaceae has become an interesting target for microbiome identification. In this work, we identified bacterial and fungal communities from the roots and surrounding soil of orchids from five heavy metal contaminated areas of the mining sites Fe deposit Sirk (C. longifolia and E. pontica), Pb-Zn deposit Jasenie (E. atrorubens and P. bifolia), and Ni-Co deposit Dobšiná (E. atrorubens). To sum up, slight deviations in $\mathrm{pH}$ of the initial soils do not significantly affect the presence of fungi and bacteria and thus the presence of the studied orchids in these localities. Similarly, the toxic elements (e.g., $\mathrm{As}, \mathrm{Pb}, \mathrm{Cr}, \mathrm{Ni}, \mathrm{Co}, \mathrm{Cu}, \mathrm{Fe}$ ), in the studied localities, do not affect the occurrence of fungi and bacteria, and also orchids. Furthermore, the study of selected factors influencing the occurrence of orchids showed that the most important factors of the occurrence of orchids in technogenic soils are the presence of a sufficient number of fungi and bacteria, sufficient light and the absence of other plant species in the habitat. Moreover, microbial communities also provide a huge benefit for orchids to be able to grow in these polluted areas. Additionally, some of these microbial communities possess huge biotechnological potential in bioremediation of heavy metal 
contaminated areas. However, to fully understand the microbiota and its interaction with orchids in heavy metal contaminated areas, further investigation is needed.

Author Contributions: Conceptualization, D.O.; methodology, D.O. and T.S.; software, J.B.; validation, M.B.; formal analysis, M.L. and M.B.; investigation, D.O. and M.B.; resources, D.O. and T.S.; data curation, J.B. and M.B.; writing_-original draft preparation, M.R. and M.B.; writing — review and editing, D.O. and T.S.; visualization, M.B.; supervision, D.O. and T.S.; project administration, D.O.; funding acquisition, D.O. and T.S. All authors have read and agreed to the published version of the manuscript.

Funding: This research was funded by the Slovak Research and Development Agency (APVV-0375-12 and APVV-16-0264).

Conflicts of Interest: The authors declare no conflict of interest.

\section{References}

1. Taylor, D.L.; Bruns, T.D. Independent, specialized invasion of ectomycorrhizal mutualism by two nonphotosynthetic orchids. Proc. Natl. Acad. Sci. USA 1997, 94, 4510-4515. [CrossRef] [PubMed]

2. McCormick, M.K.; Whigham, D.F.; O'Neill, J.P. Mycorrhizal diversity in photosynthetic terrestrial orchids. New Phytol. 2004, 163, 425-438. [CrossRef]

3. Gill, D.; Otte, D.; Endler, J.A. Fruiting failure, pollinator inefficiency and speciation in orchids, Speciation and Its Consequences. In Speciation and Its Consequences; Otte, D., Endler, J.A., Eds.; Sinauer Associates: Sunderland, MA, USA, 1989; pp. 458-481.

4. Otero, J.T.; Flanagan, N.S. Orchid diversity-Beyond deception. Trends Ecol. Evol. 2006, 21, 64-65. [PubMed]

5. Banásová, V. Vegetation of copper and antimony mine heaps. Biol. Práce 1976, 22, 1-109.

6. Rasmussen, H.N. Terrestrial Orchids: From Seed to Mycotrophic Plant; Cambridge University Press: Cambridge, UK, 1995; p. 433.

7. George, E.; Häussler, K.U.; Vetterlein, D.; Gorgus, E.; Marschner, H. Water and nutrient translocation by hyphae of Glomus mosseae. Can. J. Bot. 1992, 70, 2130-2137. [CrossRef]

8. Jasper, D.A.; Abbott, L.K.; Robson, A.D. Hyphae of a vesicular-arbuscular mycorrhizal fungus maintain infectivity in dry soil, except when the soil is disturbed. New Phytol. 1989, 112, 101-107. [CrossRef]

9. Thomas, R.S.; Franson, R.L.; Bethlenfalvay, G.J. Separation of arbuscular mycorrhizal fungus and root effect on soil aggregation. Soil Sci. Soc. Am. J. 1993, 57, 77-81. [CrossRef]

10. Ashida, J. Adaptation of fungi to metal toxicants. Annu. Rev. Phytopathol. 1965, 3, 153-174. [CrossRef]

11. Fourest, E.; Roux, J.C. Heavy Metal Biosorption by Fungal Mycelial By-Products Mechanisms and Influence of pH. Appl. Microbiol. Biotechnol. 1992, 37, 399-403. [CrossRef]

12. Hamilton, W.A. Microbially influenced corrosion as a model system for the study of metal microbe interactions: A unifying electron transfer hypothesis. Biofouling 2003, 19, 65-76. [CrossRef]

13. Remenár, M.; Harichová, J.; Zámocký, M.; Pangallo, D.; Szemes, T.; Budiš, J.; Šoltys, K.; Ferianc, P. Metagenomics of a nickel-resistant bacterial community in an anthropogenic nickel-contaminated soil in southwest Slovakia. Biologia 2017, 72, 971-981. [CrossRef]

14. Caporaso, J.G.; Lauber, C.L.; Walters, W.A.; Berg-Lyons, D.; Huntley, J.; Fierer, N.; Owens, S.M.; Betley, J.; Fraser, L.; Bauer, M.; et al. Ultra-high-throughput microbial community analysis on the Illumina HiSeq and MiSeq platforms. ISME J. 2012, 6, 1621-1624. [CrossRef]

15. Fadrosh, D.W.; Ma, B.; Gajer, P.; Sengamalay, N.; Ott, S.; Brotman, R.M.; Ravel, J. An improved dual-indexing approach for multiplexed 16S rRNA gene sequencing on the Illumina MiSeq platform. Microbiome 2014, 2, 1-7. [CrossRef] [PubMed]

16. Kraková, L.; Šoltys, K.; Otlewska, A.; Pietrzak, K.; Purkrtová, S.; Savická, D.; Puškárová, A.; Bučková, M.; Szemes, T.; Budiš, J.; et al. Comparison of methods for identification of microbial communities in book collections: Culture-dependent (sequencing and MALDI-TOF MS) and culture-independent (Illumina MiSeq). Int. Biodeterior. Biodegrad. 2018, 131, 51-59. [CrossRef]

17. Böhmer, M.; Smol'ak, D.; Ženišová, K.; Čaplová, Z.; Pangallo, D.; Puškárová, A.; Bučková, M.; Cabicarová, T.; Budiš, J.; Šoltýs, K.; et al. Comparison of the Microbial Diversity During Two Different Wine Fermentation Processes. Appl. Sci. 2020, 367, fnaa150. [CrossRef] 
18. Hong, C.; Si, Y.; Xing, Y.; Li, Y. Illumina MiSeq sequencing investigation on the contrasting soil bacterial community structures in different iron mining areas. Environ. Sci. Pollut. Res. 2015, 22, 10788-10799. [CrossRef]

19. Xiao, E.Z.; Krumins, V.; Tang, S.; Xiao, T.F.; Ning, Z.P.; Lan, X.L.; Sun, W.M. Correlating microbial community profiles with geochemical conditions in a watershed heavily contaminated by an antimony tailing pond. Environ. Pollut. 2016, 215, 141-153. [CrossRef]

20. Račko, M.; Ozdín, D.; Kučerová, G.; Jurkovič, L.; Vaculík, M. Occurrence and uptake of heavy metals by selected terrestrial orchids in extreme conditions of initial soils on previous mining sites. Biologia 2020, in press. [CrossRef]

21. Ďud'a, R.; Ozdín, D. Minerály Slovenska; Granit: Praha, Czech Republic, 2012; 480p.

22. Števko, M.; Ozdín, D. Supergene native silver and acanthite from the Jasenie-Soviansko base metals deposit, Nízke Tatry Mts. (Slovak Republic). Bull. Mineral. Petrolog. Odd. Nár. Muz. 2012, 20, 47-51.

23. Grecula, P. (Ed.) Mineral Deposits of the Slovak Ore Mountains; Geocomplex: Bratislava, Slovakia, 1995; Volume 1, 834p.

24. Cao, L.; Qiu, Z.; Dai, X.; Tan, H.; Lin, Y.; Zhou, S. Isolation of Endophytic Actinomycetes From Roots and Leaves of Banana (Musa Acuminata) Plants and Their Activities Against Fusarium oxysporumf. sp. cubense. World J. Microbiol. Biotechnol. 2004, 20, 501-504. [CrossRef]

25. Klindworth, A.; Pruesse, E.; Schweer, T.; Peplies, J.; Quast, C.; Horn, M.; Glöckner, F.O. Evaluation of general $16 \mathrm{~S}$ ribosomal RNA gene PCR primers for classical and next-generation sequencing-based diversity studies. Nucleic Acids Res. 2013, 41, e1. [CrossRef] [PubMed]

26. White, T.J.; Bruns, T.; Lee, S.; Taylor, J. Amplification and direct sequencing of fungal ribosomal RNA genes for phylogenetics. In PCR Protocols: A Guide to Methods and Applications; Innis, M.A., Gelfand, D.H., Sninsky, J.J., White, T.J., Eds.; Academic Press: New York, NY, USA, 1990; pp. 315-321.

27. Bolger, A.M.; Lohse, M.; Usadel, B. Trimmomatic: A flexible trimmer for Illumina sequence data. Bioinformatics 2014, 30, 2114-2120. [CrossRef] [PubMed]

28. Andrews, S. FastQC: A Quality Control Tool for High Throughput Sequence Data. 2010. Available online: http://www.bioinformatics.babraham.ac.uk/projects/fastqc (accessed on 20 November 2015).

29. Zhang, J.; Kobert, K.; Flouri, T.; Stamatakis, A. PEAR: A fast and accurate Illumina Paired-End reAd mergeR. Bioinformatics 2014, 30, 614-620. [CrossRef]

30. Bolyen, E.; Rideout, J.R.; Dillon, M.R.; Bokulich, N.A.; Abnet, C.C.; Al-Ghalith, G.A.; Alexander, H.; Alm, E.J.; Arumugam, M.; Asnicar, F.; et al. Reproducible, interactive, scalable and extensible microbiome data science using QIIME 2. Nat. Biotechnol. 2019, 37, 852-857. [CrossRef]

31. Rognes, T.; Flouri, T.; Nichols, B.; Quince, C.; Mahé, F. VSEARCH: A versatile open source tool for metagenomics. PeerJ 2016, 4, e2584. [CrossRef] [PubMed]

32. Pedregosa, F.; Varoquaux, G.; Gramfort, A.; Michel, V.; Thirion, B.; Grisel, O.; Blondel, M.; Prettenhofer, P.; Weiss, R.; Dubourg, V.; et al. Scikit-learn: Machine Learning in Python. J. Mach. Learn. Res. 2012, 12, 2825-2830.

33. Quast, C.; Pruesse, E.; Yilmaz, P.; Gerken, J.; Schweer, T.; Yarza, P.; Peplies, J.; Glöckner, F.O. The SILVA ribosomal RNA gene database project: Improved data processing and web-based tools. Nucleic Acids Res. 2013, 41, D590-D596. [CrossRef]

34. Nilsson, R.H.; Larsson, K.H.; Taylor, A.F.S.; Bengtsson-Palme, J.; Jeppesen, T.S.; Schigel, D.; Kennedy, P.; Picard, K.; Glöckner, F.O.; Tedersoo, L.; et al. The UNITE database for molecular identification of fungi: Handling dark taxa and parallel taxonomic classifications. Nucleic Acids Res. 2019, 47, D259-D264. [CrossRef]

35. Faith, D.P. Conservation evaluation and phylogenetic diversity. Biol. Conserv. 1992, 61, 1-10. [CrossRef]

36. Budiš, J.; Krampl, W.; Kucharík, M.; Hekel, R.; Lichvár, M.; Smol’ak, D.; Böhmer, M.; Baláž, A.; Ďuriš, F.; Gazdarica, J.; et al. SnakeLines: Integrated set of computational pipelines for paired-end sequencing reads. 2019. Available online: https://github.com/jbudis/snakelines/ (accessed on 18 October 2019).

37. Köster, J.; Rahmann, S. Snakemake—S scalable bioinformatics workflow engine. Bioinformatics 2012, 28, 2520-2522. [CrossRef]

38. Turnau, K.; Gawroński, S.; Ryszka, P.; Zook, D. Mycorrhizal-based phytostabilization of Zn-Pb tailings: Lessons from the Trzebionka mining works (Southern Poland). In Bio-Geo Interactions in Metal-Contaminated Soils; Soil Biology; Varma, A., Kothe, E., Eds.; Springer: Berlin, Germany, 2012; Volume 31, pp. 327-348. 
39. Punz, W.; Sieghardt, H. The response of roots of herbaceous plant species to heavy metals. Environ. Exp. Bot. 1993, 33, 85-98. [CrossRef]

40. Rufo, L.; de la Fuente, V. Successional dynamics of the climatophile vegetation of the mining territory of the Río Tinto basin (Huelva, Spain): Soil characteristics and implications for phytoremediation. Arid Land Res. Manag. 2010, 24, 301-327. [CrossRef]

41. Dearnaley, J.D.W.; Martos, F.; Selosse, M.A. Orchid mycorrhizas: Molecular ecology, physiology, evolution and conservation aspects. In Fungal Associations, 2nd ed.; The Mycota (9); Hock, B., Ed.; Springer-Verlag: Berlin, Germany, 2012; pp. 207-230.

42. Dearnaley, J.D.W. Further advances in orchid mycorrhizal research. Mycorrhiza 2007, 17, 475-486. [CrossRef] [PubMed]

43. Shefferson, R.P.; Kull, T.; Tali, K. Mycorrhizal interactions of orchids colonizing Estonian mine tailings hills. Am. J. Bot. 2008, 95, 156-164. [CrossRef] [PubMed]

44. Ranjan, R.; Rani, A.; Metwally, A.; McGee, H.S.; Perkins, D.L. Analysis of the microbiome: Advantages of whole genome shotgun versus $16 \mathrm{~S}$ amplicon sequencing. Biochem. Biophys. Res. Commun. 2016, 469, 967-977. [CrossRef]

45. Knight, R.; Vrbanac, A.; Taylor, B.C.; Aksenov, A.; Callewaert, C.; Debelius, J.; Gonzalez, A.; Kosciolek, T.; McCall, L.I.; McDonald, D.; et al. Best practices for analysing microbiomes. Nat. Rev. Microbiol. 2018, 16, 410-422. [CrossRef]

46. Quince, C.; Walker, A.W.; Simpson, J.T.; Loman, N.J.; Segata, N. Shotgun metagenomics, from sampling to analysis. Nat. Biotechnol. 2017, 35, 833-844. [CrossRef]

47. Blagodatskaya, E.V.; Anderson, T.H. Interactive effects of $\mathrm{pH}$ and substrate quality on the fungal-to-bacterial ratio and $\mathrm{QCO}_{2}$ of microbial communities in forest soils. Soil Biol. Biochem. 1998, 30, 1269-1274. [CrossRef]

48. Fierer, N.; Jackson, R.B. The diversity and biogeography of soil bacterial communities. Proc. Natl. Acad. Sci. USA 2006, 103, 626-631. [CrossRef]

49. Stępniewska, H.; Uzarowicz, Ł.; Błońska, E.; Kwasowski, W.; Słodczyk, Z.; Gałka, D.; Hebda, A. Fungal abundance and diversity as influenced by properties of Technosols developed from mine wastes containing iron sulphides: A case study from abandoned iron sulphide and uranium mine in Rudki, south-central Poland. Appl. Soil Ecol. 2020, 145, 103349. [CrossRef]

50. Šimonovičová, A.; Kraková, L.; Pauditšová, E.; Pangallo, D. Occurrence and diversity of cultivable autochthonous microscopic fungi in substrates of old environmental loads from mining activities in Slovakia. Ecotoxicol. Environ. Saf. 2019, 172, 194-202. [CrossRef] [PubMed]

51. Jurkiewicz, A.; Turnau, K.; Mesjasz-Przybyłowicz, J.; Przybyłowicz, W.; Godzik, B. Heavy metal localisation in mycorrhizas of Epipactis atrorubens (Hoffm.) Besser (Orchidaceae) from zinc mine tailings. Protoplasma 2001, 218, 117-124. [CrossRef] [PubMed]

52. Šimonovičová, A.; Ferianc, P.; Vojtková, H.; Pangallo, D.; Hanajík, P.; Kraková, L.; Feketeová, Z.; Čerňanský, S.; Okenicová, L.; Žemberyová, M.; et al. Alkaline Technosol contaminated by former mining activity and its culturable autochthonous microbiota. Chemosphere 2017, 171, 89-96. [CrossRef]

53. Timling, I.; Taylor, D.L. Peeking through a frosty window: Molecular insights into the ecology of Arctic soil fungi. Fungal Ecol. 2012, 5, 419-429. [CrossRef]

54. Chávez, R.; Fierro, F.; García-Rico, R.O.; Vaca, I. Filamentous fungi from extreme environments as a promising source of novel bioactive secondary metabolites. Front. Microbiol. 2015, 6, 903. [CrossRef]

55. Gupta, H.; Kumar, R.; Park, H.S.; Jeon, B.H. Photocatalytic efficiency of iron oxide nanoparticles for the degradation of priority pollutant anthracene. Geosyst. Eng. 2017, 20, 21-27. [CrossRef]

56. Mohammadi, K.; Khalesro, S.; Sohrabi, Y.; Heidari, G. A review: Beneficial effects of the mycorrhizal fungi for plant growth. J. Appl. Environ. Biol. Sci. 2011, 1, 310-319.

57. Baldrian, P. Interactions of heavy metals with white-rot fungi. Enzym. Microb. Technol. 2003, 32, 78-91. [CrossRef]

58. Zhang, L.; Chen, J.; Gao, C.; Guo, S. Mycena sp., a mycorrhizal fungus of the orchid Dendrobium officinale. Mycol. Prog. 2012, 11, 395-401. [CrossRef] 
59. Cui, Z.; Zhang, X.; Yang, H.; Sun, L. Bioremediation of heavy metal pollution utilizing composite microbial agent of Mucor circinelloides, Actinomucor sp. and Mortierella sp. J. Environ. Chem. Eng. 2017, 5, 3616-3621. [CrossRef]

60. Niu, H.; Xu, X.S.; Wang, J.H.; Volesky, B. Removal of lead from aqueous solutions by Penicillium biomass. Biotechnol. Bioeng. 1993, 42, 785-787. [CrossRef] [PubMed]

61. Chai, B.; Wu, Y.; Liu, P.; Liu, B.; Gao, M. Isolation and phosphate-solubilizing ability of a fungus, Penicillium sp. from soil of an alum mine. J. Basic Microbiol. 2011, 51, 5-14. [CrossRef]

62. Mishra, S.; Chaudhury, G. Biosorption of Copper by Penicillium sp. Int. J. 1995, 14, 111-126. [CrossRef]

63. Gadd, G.M. Metals, Minerals and Microbes: Geomicrobiology and Bioremediation. Microbiology 2010, 156, 609-643. [CrossRef]

64. Feng, G.; Xie, T.; Wang, X.; Bai, J.; Tang, L.; Zhao, H.; Wei, W.; Wang, M.; Zhao, Y. Metagenomic analysis of microbial community and function involved in cd-contaminated soil. BMC Microbiol. 2018, 18, 11. [CrossRef]

65. Guo, H.; Nasir, M.; Lv, J.; Dai, Y.; Gao, J. Understanding the variation of microbial community in heavy metals contaminated soil using high throughput sequencing. Ecotoxicol. Environ. Saf. 2017, 144, 300-306. [CrossRef]

66. Marcin, C.; Marcin, G.; Justyna, M.P.; Katarzyna, K.; Maria, N. Diversity of microorganisms from forest soils differently polluted with heavy metals. Appl. Soil Ecol. 2013, 64, 7-14.

67. Tipayno, S.C.; Truu, J.; Samaddar, S.; Truu, M.; Preem, J.K.; Oopkaup, K.; Espenberg, M.; Chatterjee, P.; Kang, Y.; Kim, K.; et al. The bacterial community structure and functional profile in the heavy metal contaminated paddy soils, surrounding a nonferrous smelter in South Korea. Ecol. Evol. 2018, 8, 6157-6168. [CrossRef]

68. Ma, Y.; Wang, Y.; Chen, Q.; Li, Y.; Guo, D.; Nie, X.; Peng, X. Assessment of heavy metal pollution and the effect on bacterial community in acidic and neutral soils. Ecol. Indic. 2020, 117, 106626. [CrossRef]

69. Brewer, T.E.; Handley, K.M.; Carini, P.; Gilbert, J.A.; Fierer, N. Genome reduction in an abundant and ubiquitous soil bacterium 'Candidatus Udaeobacter copiosus'. Nat. Microbiol. 2016, 2, 16198. [CrossRef]

70. Glöckner, F.O.; Kube, M.; Bauer, M.; Teeling, H.; Lombardot, T.; Ludwig, W.; Gade, D.; Beck, A.; Borzym, K.; Heitmann, K.; et al. Complete genome sequence of the marine planctomycete Pirellula sp. strain 1. Proc. Natl. Acad. Sci. USA 2003, 100, 8298-8303. [CrossRef] [PubMed]

71. Turner, T.R.; James, E.K.; Poole, P.S. The plant microbiome. Genome Biol. 2013, 14, 1267-1273. [CrossRef] [PubMed]

72. Yasouri, F.N. Plasmid mediated degradation of diazinon by three bacterial strains Pseudomonas sp., Flavobacterium sp. and Agrobacterium sp. Asian J. Chem. 2006, 18, 2437-2444.

73. Lakshmi, C.V.; Kumar, M.; Khanna, S. Biotransformation of chlorpyrifos and bioremediation of contaminated soil. Int. Biodeter. Biodegr. 2008, 62, 204-209. [CrossRef]

74. Sun, W.; Dong, Y.; Gao, P.; Fu, M.; Ta, K.; Li, J. Microbial communities inhabiting oil-contaminated soils from two major oilfields in Northern China: Implications for active petroleum-degrading capacity. J. Microbiol. 2015, 53, 371-378. [CrossRef]

75. Chaudhary, D.K.; Kim, J. Sphingomonas naphthae sp. nov., isolated from oil-contaminated soil. Int. J. Syst. Evol. Microbiol. 2016, 66, 4621-4627. [CrossRef]

76. Chen, C.; Liu, Q.; Liu, C.; Yu, J. Effect of different enrichment strategies on microbial community structure in petroleum-contaminated marine sediment in Dalian, China. Mar. Pollut. Bull. 2017, 117, 274-282. [CrossRef] [PubMed]

77. Zhao, L.; Zhang, C.; Li, H.; Bao, M.; Sun, P. Regulation of different electron acceptors on petroleum hydrocarbon biotransformation to final products in activated sludge biosystems. Bioprocess Biosyst. Eng. 2019, 42, 643-655. [CrossRef]

78. Feng, X.; Liu, Z.; Jia, X.; Lu, W. Distribution of Bacterial Communities in Petroleum-Contaminated Soils from the Dagang Oilfield, China. Trans. Tianjin Univ. 2020, 26, 22-32. [CrossRef]

79. Elshahed, M.S.; Youssef, N.H.; Luo, Q.; Najar, F.Z.; Roe, B.A.; Sisk, T.M.; Bühring, S.I.; Hinrichs, K.U.; Krumholz, L.R. Phylogenetic and Metabolic Diversity of Planctomycetes from Anaerobic, Sulfide- and Sulfur-Rich Zodletone Spring, Oklahoma. Appl. Environ. Microbiol. 2007, 73, 4707-4716. [CrossRef] [PubMed] 
80. Arp, D.; Sayavedra-Soto, L.; Hommes, N. Molecular biology and biochemistry of ammonia oxidation by Nitrosomonas europaea. Arch. Microbiol. 2002, 178, 250-255. [CrossRef] [PubMed]

81. Coombs, J.T.; Franco, C.M.M. Isolation and identification of actinobacteria isolated from surface-sterilized wheat roots. Appl. Environ. Microbiol. 2003, 69, 5603-5608. [CrossRef] [PubMed]

82. Chaudhary, D.K.; Kim, J. Flavobacterium olei sp. nov., a novel psychrotolerant bacterium isolated from oil-contaminated soil Free. Int. J. Syst. Evol. Microbiol. 2017, 67, 2211-2218. [CrossRef] [PubMed]

Publisher's Note: MDPI stays neutral with regard to jurisdictional claims in published maps and institutional affiliations.

(C) 2020 by the authors. Licensee MDPI, Basel, Switzerland. This article is an open access article distributed under the terms and conditions of the Creative Commons Attribution (CC BY) license (http://creativecommons.org/licenses/by/4.0/). 